\title{
Correspondence
}

\section{Synacthen test}

SIR-I have read with considerable interest the paper by Greig et al. (Postgrad. med. J., 1969, 45, 307) describing their criteria for distinguishing normal from subnormal adrenocortical function using the 30-min Synacthen test, but I am not convinced that this test is a reliable screening procedure for the detection or exclusion of adrenocortical failure. The data in this paper was originally presented at a symposium on the investigation of hypothalamic-pituitary-adrenal function at the Royal Society of Medicine in February 1967, and in the discussion which followed Bayliss (1968) reported instances of patients who failed to respond to this test but who subsequently demonstrated a striking adrenal response to more prolonged stimulation. This is hardly surprising for Synacthen has a relatively short duration of action and is a weak stimulus to the adrenal glands compared with other ACTH preparations.

In the authors' control subjects the mean rise in the plasma 11-hydroxycorticoid levels $30 \mathrm{~min}$ after Synacthen was only $15.5 \mu \mathrm{g} / 100 \mathrm{ml}$, compared with a mean normal rise of $24.7 \mu \mathrm{g} / 100 \mathrm{ml} 1 \mathrm{hr}$ after an intramuscular injection of 25 units of the natural hormone (Maynard et al., 1966), and a mean normal rise of $44.8 \mu \mathrm{g} / 100 \mathrm{ml} 5 \mathrm{hr}$ after a single intramuscular injection of ACTH gel (Mattingly, 1968). I agree that a normal response to Synacthen will exclude Addison's disease, for in these patients the adrenal remnant is already being maximally stimulated by endogenous corticotrophin, but does a subnormal response necessarily indicate a significant degree of secondary adrenal atrophy when the stimulus to the adrenal glands is of such a brief duration? Greig and his colleagues have attempted to answer this by comparing the results obtained by the Synacthen test in three groups of patients with those obtained following a 4-hr intravenous infusion of porcine corticotrophin. Unfortunately, they do not give their criteria for a normal response to the latter stimulus, but it would appear from their Fig. 2 that the response of their ten steroid treated patients with a subnormal Synacthen response was significantly less than that obtained in the steroid treated patients with a normal Synacthen result and in their normal controls. However, if one takes two standard deviations on either side of the means to indicate the range of observations it would appear that there must have been some overlap between these three groups, and I find it difficult to accept their statement that there were no false positive or false negative diagnoses of adreno- cortical insufficiency when using the Synacthen test.

The authors emphasize that the Synacthen test is primarily a screening procedure and that a subnormal response allows only a provisional diagnosis of adrenocortical insufficiency. If further investigations are necessary to confirm the diagnosis and to make the distinction between primary and secondary adrenal disease why not use them from the beginning? The rise in the plasma 11-hydroxycorticoid level $5 \mathrm{hr}$ after the intramuscular injection of a depot preparation of ACTH is a much more reliable measure of the patient's adrenal reserve, and Synacthen depot can be used instead of ACTH gel or ACTH/CMC in patients known to be, or likely to be sensitized to the natural hormone. Even this stimulus may be insufficient to differentiate between primary and secondary adrenal failure but this distinction can be readily made by repeating the depot injections twice a day for at least 3 days. Since the synthetic analogues of cortisol and cortisone do not fluoresce, the patient can be covered during this procedure until the results are known.

\section{Royal Devon and Exeter Hospital,} Exeter

D. Mattingly

\section{References}

Bayliss, R.I.S. (1968) Mem. Soc. Endocr. 17, 189.

MatTingly, D. (1968) Fourth Symposium on advanced Medicine (Ed. by O. Wrong), p. 158. Pitman, London.

MaYNARD, D.E., FolK, R.L., Riley, T.R., Wieland, R.G. Gwinup, G. \& HaMwi, G.J. (1966) Ann. intern. Med. 64, 552.

We have shown this letter to Dr. Grey, who replies as follows:

SIR-Dr Mattingly's comments on our paper are fair and reasonable but we would like to reply to the various points he raises.

We have felt from the inception of this study that if we clinicians are to use the Synacthen test in general ward practice then we are obliged, by appropriate collation of data, to draw up some workable criteria on which to base our decisions as to the likelihood of normality of adrenocortical function in the individual patient.

We agree, and state it in our paper, that the Synacthen test is only a screening test. We feel that, if the response is normal then adrenal failure is excluded; but if the response is subnormal then 
adrenal failure should be seriously considered but not accepted as a definitive diagnosis until proven by other procedures such as more prolonged corticotrophin stimulation. Since the large majority of patients tested by all procedures for suspected adrenal insufficiency are found not to have this, there seems to be a place for the simple screening test like the Synacthen test. As Dr Mattingly says in his letter ' $I$ agree that a normal response to Synacthen will exclude Addison's disease'; it is quite probable that doctors will sometimes find subnormal responses to the Synacthen test and subsequently normal responses to more powerful adrenal stimulation in the same patients. We have not, however, so far experienced this if proper care is taken in giving the Synacthen.

Our normal range of responses to the corticotrophin infusions was the data obtained in the control group of patients shown in Fig. 2. Dr Mattingly disputes our claim that there were no false positive or false negative diagnoses; our discrimination arises we think, because we are using three different cortisol values to make our decisions (see Table 1).

We feel that, in ill patients when a quick therapeutic decision may have to be made, the $30-\mathrm{min}$ Synacthen test may be employed before hydrocortisone is given but that the 5-hr infusion test mentioned by $\mathrm{Dr}$ Mattingly is too prolonged. Although ill patients can have proper testing of adrenal function performed after recovery, it is helpful to have some index of this before hydrocortisone is given. Thus, by and large, we are not in dispute with Dr Mattingly. Perhaps the chief value of our paper is to stimulate critical thought amongst those who do use the Synacthen test. We would suggest that all clinicians adopting this test should keep careful records and report their experience.

University Department of Medicine,

W. R. GREIG AND Royal Infirmary, Glasgow CO-WORKERS 